\title{
Plasmodium vivax Mimicking Morphologic Features of Plasmodium falciparum
}

\author{
Najia Ghanchi ${ }^{1}$, Adnan A. Khan ${ }^{2}$, Ahmed Raheem ${ }^{3}$, Mohammad A. Beg ${ }^{1}$ \\ 1. Pathology, Aga Khan University Hospital, Karachi, PAK 2. Anesthesiology, Aga Khan University Hospital, Karachi, \\ PAK 3. Emergency Medicine, Aga Khan University Hospital, Karachi, PAK
}

Corresponding author: Mohammad A. Beg, masim.beg@aku.edu

\begin{abstract}
Plasmodium vivax (P. vivax) is the most common cause of malaria in Pakistan. Several cases of severe malaria due to P.vivax have been reported from Pakistan and India, however morphological characteristics of the parasite have been mainly ignored. We present two cases of P. vivax mono-infection, which were characterized by multiple infected red blood cells, similar to that seen in Plasmodium falciparum, as observed under microscopy. Both cases were confirmed as mono-infection of P.vivax on Giemsa stained thick and thin films, malaria rapid diagnostic test (RDT) and Polymerase Chain Reaction (PCR). Morphology on peripheral blood smear remains the gold standard for diagnosis of malaria and mimicking morphological features leads to misdiagnosis and mismanagement of patients.
\end{abstract}

Categories: Pathology, Infectious Disease, Other

Keywords: plasmodium vivax, mimicking, p.falciparum, throphozoite

\section{Introduction}

Plasmodium vivax (P. vivax) is the most common cause of malaria in Pakistan and contributes significantly to worldwide morbidity and mortality. An estimated 2.5 billion people are currently at the risk of P. vivax transmission, with approximately $91 \%$ of this population located in central and Southeast Asia [1]. In Pakistan, malaria remains an endemic disease with an estimated health care burden of 1.6 million cases annually, of which, $64 \%$ of cases can be attributed to P. vivax [2].

Review began 09/25/2020 Review ended 11/06/2020 Published 11/09/2020

(c) Copyright 2020 Ghanchi et al. This is an open access article distributed under the terms of the Creative Commons Attribution License CC-BY 4.0., which permits unrestricted use, distribution, and reproduction in any medium, provided the original author and source are credited.
Although typically regarded as benign, P. vivax has the potential to cause severe disease. In such cases, the clinical profile often mimics that of $P$. falciparum malaria with renal failure, cerebral malaria and hematologic abnormalities as prominent features [3]. Apart from clinical features, morphological features of P. vivax can also resemble that of P. falciparum. Morphology remains the gold standard for diagnosis of malaria [4]. This may lead to misdiagnosis followed by mismanagement, especially in resources limited areas where other diagnostic modalities like rapid diagnostic test (RDT) and Polymerase Chain Reaction (PCR) are unavailable. Herein, we present two cases where P. vivax was seen to imitate Plasmodium falciparum both clinically and morphologically.

\section{Case Presentation}

\section{Case 1}

A 57-year-old male presented to the Emergency Department of the Aga Khan University Hospital (AKUH) with a one-day history of fever. The fever was intermittent, associated with chills, rigours, cough, vomiting, watery diarrhoea and progressive drowsiness. His vitals included a temperature of 38.5-degree celsius, tachycardia with a pulse of $105 /$ minute, blood pressure $90 / 45 \mathrm{mmHg}$, tachypnea with respiratory rate $25 /$ minute and oxygen saturation was maintained at $98 \%$. On examination, the patient was drowsy but oriented in time, place. He had conjunctival pallor and appeared dehydrated. Respiratory examination revealed bilateral basal crepitation's. Cardiovascular and abdominal examinations were within normal limits. The neurological assessment revealed a Glasgow Coma Score (GCS) score of 13/15. His pupils were reactive to light but anisocoric (Left pupil 3mm, Right pupil $2 \mathrm{~mm}$ ). Motor examination showed right up to going plantar.

The patient's arterial blood gases revealed a normal pH of 7.41 but decreased levels of $\mathrm{CO}_{2}(20.30 \mathrm{mmHg}), \mathrm{O}_{2}$ (73.80 $\mathrm{mmHg}$ ) and bicarbonate (12.5 mmHg). Low values of $\mathrm{Hb} 8.2$, white cell count (WCC) normal and low platelets 110 with treatment these became within normal limits.

Results of CBC showed low $\mathrm{Hb}$ and low platelets as above, and peripheral film, thick and thin film morphology should be added Thick and Thin blood films showed trophozoites and gametocytes of P.vivax, in addition to immuno-chromatography test (ICT) and PCR confirmation of mono-infection with P. vivax. Serum lactate was raised at $7.3 \mathrm{mmol} / \mathrm{L}$.

Coagulation profile revealed an elevated prothrombin time (PT) of 35.4 seconds, a raised INR of 3.42 and an 
elevated Activated Partial Thromboplastin Time (APTT) of more than 120 seconds. Liver function tests revealed total, direct and indirect bilirubin $2.8 \mathrm{mg} / \mathrm{dL}, 2.4 \mathrm{mg} / \mathrm{dL}$ and $0.4 \mathrm{mg} / \mathrm{dL}$ respectively. Liver enzymes were within normal. Dengue antigen was negative. Malaria ICT test was also positive for P.vivax.

Radiological studies showed normal chest X-ray and CT scan of the head while an ultrasound examination of the liver revealed a fatty liver. A provisional diagnosis of complicated malaria and acute kidney injury was made. A presumptive diagnosis of acute kidney injury was made on the abnormal Creatinine and electrolytes observed; these became within normal limits with treatment.

He was started on a broad-spectrum antibiotic (Piperacillin and Tazobactam $4.5 \mathrm{mg}$ ), and Artemisinin-based combination therapy (Artemether and Lumefantrine), an antiemetic (Metoclopramide $10 \mathrm{mg}$ ), and an antipyretic (Paracetamol $1000 \mathrm{mg}$ ). Rehydration with a normal saline infusion was started, and the patient's serum potassium ion levels were replenished. Subsequently, the patient remained vitally stable. He reported a single fever spike at 38degrees Celsius on the second day of admission followed by an uneventful hospital stay. The patient's platelet levels rose steadily to normal levels, and he was discharged on the 4 th day of admission.

A similar case of infection with P. vivax is presented below where P. vivax was seen to parasite P. falciparum in morphology as observed under the microscope. However, no atypical features of disease were observed in this patient. Both cases were confirmed mono-infection of P. vivax on PCR as well.

\section{Case 2}

A seven-year-old female, known case of dilated cardiomyopathy, was admitted to the AKUH with a threeday history of fever and increased frequency of urination. The fever was associated with rigours and chills. Her past medical history was significant for a similar episode of fever two weeks prior, which resolved with a course of oral Amoxicillin and Clavulanic acid. Patient vitals at presentation were the temperature of 38degree Celsius, tachypnea with a respiratory rate of 30 breaths/min, hypotensive with blood pressure 79/38 $\mathrm{mmHg}$, tachypnea with a heart rate of 121 beats/min and decreased oxygen saturation of $91 \%$.

On examination, the patient appeared lean for age $(18 \mathrm{~kg})$ but alert and oriented in time and place. She had conjunctival pallor. Cardiovascular, respiratory and abdominal examinations were within normal limits. The neurological assessment revealed a GCS score of $15 / 15$. The rest of the examination was unremarkable.

During the hospital stay, the patient remained febrile and developed thrombocytopenia. Based on clinical symptoms and positive malaria parasite on Giemsa stained blood smears and ICT test, a diagnosis of P. vivax malaria was made. The patient was administered IV Ceftriaxone and Artemisinin-based combination therapy (Artemether and Lumefantrine). The patients' platelet count rose to normal levels, and she was discharged.

Morphological features of the Giemsa stained slides from two cases 1 (A-C) and 2 (A-C) which were similar to P. falciparum morphologically showing multiple infected RBCs as presented in figure 1 . 


\section{Cureus}
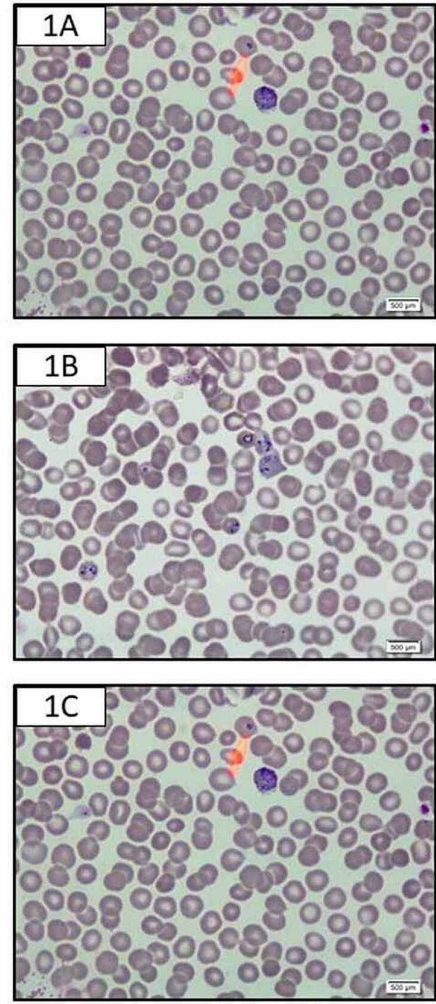
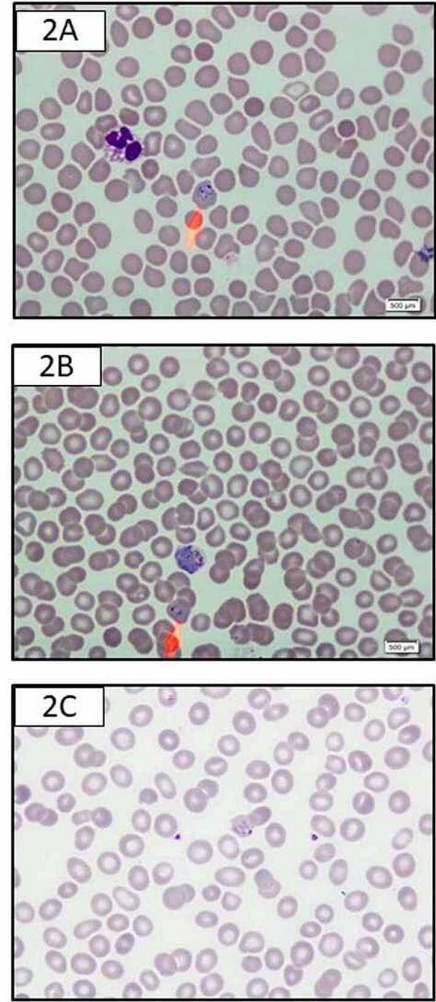

FIGURE 1: Morphological features of the giemsa stained slides from two cases $1(A-C)$ and $2(A-C)$ which were similar to P.falciparum morphologically showing multiple infected RBCs

Plasmodium Falciparum ( P.falciparum), Red blood cells (RBCs)

\section{Discussion}

P. vivax malaria is commonly associated with chills, vomiting, malaise, headache, fever and myalgia. Recently, however, P. vivax infection has been documented to present as severe malaria previously attributed solely to P. falciparum [5]. The spectrum of clinical manifestations exhibited by P.vivax malaria suggests an interplay between host, parasite and external factors [6-7]. Among these, parasite virulence appears to play an evolving role. Anti-malarial drug resistance, invasion of Duffy negative erythrocytes, production of anti-erythrocyte antibodies, and P. vivax polymorphisms altering cytokine production emphasize the evolution of parasite virulence [8-11]. These observations beg the question of whether morphological similarities exist between P. falciparum and 'malignant' P. vivax strains, in addition to similarities in pathophysiology and clinical manifestations. Furthermore, more studies are needed to ascertain whether P. vivax strains causing severe malaria differ morphologically from those causing 'benign' disease.

Peripheral blood smear examination is the gold standard for the diagnosis of malaria. For that purpose, we use thick and thin Giemsa-stained blood smears to identify the malarial parasites. Schizonts, trophozoites and gametocytes are commonly seen stages of the malarial parasite; while, cases with an atypical morphology of P. vivax have also been observed.

Recent studies have also suggested similarity in morphology between the Plasmodium species themselves. An imported case from Gabon has revealed up to 6 amoeboid trophozoites in a single red blood cell as well as morphologically abnormal intra-erythrocytic stages of P. vivax that resemble P. ovale [12]. Overlapping morphological features between P. vivax and P. ovale have also been reported from Spain [13]. Furthermore, one study reports atypical falciparum malaria imported from Africa, whose blood smear contained many large trophozoites, with punctiform or massive brown pigment granules; the body shape of this plasmodium specimen was similar to that of P. vivax and P. ovale [14].

Recognition of such atypical morphology of P. vivax is important to avoid diagnostic confusion with spirochetes, trypanosomes and other plasmodia species and to guide effective management and treatment of patients. 


\section{Conclusions}

This study can serve as a basis for future studies discussing atypical morphological features of P. vivax and correlating them to the severity of the disease. Should such a correlation be found, it can be used to distinguish complicated from uncomplicated malaria at an early stage to initiate appropriate early treatment and prevent further complications.

\section{Additional Information \\ Disclosures}

Human subjects: Consent was obtained by all participants in this study. Aga Khan University Hospital issued approval 4834-Pat-ERC-17. 4834-Pat-ERC-17. PI Dr Asim Beg: Laboratory-based Diagnosis of Malaria parasite (Plasmodium falciparum \& Plasmodium vivax) at Aga Khan University Hospital Thank you for your response to ERC recommendation received on August 8, 2017, regarding the above-mentioned study. Your response adequately answered the recommendation made by the Committee. The study was accepted as an exemption. You may proceed with the study. Dr Shaista Khan Chairperson Ethical Review Committee . Conflicts of interest: In compliance with the ICMJE uniform disclosure form, all authors declare the following: Payment/services info: All authors have declared that no financial support was received from any organization for the submitted work. Financial relationships: All authors have declared that they have no financial relationships at present or within the previous three years with any organizations that might have an interest in the submitted work. Other relationships: All authors have declared that there are no other relationships or activities that could appear to have influenced the submitted work.

\section{Acknowledgements}

We would like to acknowledge Naveera Khan and Aqsa Khalid with the help of administrative and scientific work.

\section{References}

1. Wassmer SC, Taylor TE, Rathod, et al.: Investigating the pathogenesis of severe malaria: a multidisciplinary and cross-geographical approach. Am J Trop Med Hyg. 2015, 93:42-56. 10.4269/ajtmh.14-0841

2. WHO. World malaria report. Switzerland: World Health Organisation. (2012). Accessed: June 6, 2020: https://www.who.int/malaria/publications/world_malaria_report_2012/en/.

3. Trampuz A, Jereb M, Muzlovic I, Prabhu RM: Clinical review: severe malaria. Crit Care. 2003, 7:315-23. 10.1186/cc2183

4. Abanyie FA, Arguin PM, Gutman J: State of malaria diagnostic testing at clinical laboratories in the United States, 2010: a nationwide survey. Malar J. 2011, 10:340. 10.1186/1475-2875-10-340

5. Naing C, Whittaker MA, Nyunt Wai V, Mak JW: Is Plasmodium vivax malaria a severe malaria?: a systematic review and meta-analysis. PLoS Negl Trop Dis. 2014, 8:3071. 10.1371/journal.pntd.0003071

6. Howes RE, Battle KE, Mendis KN, et al.: Global epidemiology of Plasmodium vivax. Am J Trop Med Hyg. 2016, 95:15-34. 10.4269/ajtmh.16-0141

7. Bassat Q, Alonso PL: Defying malaria: fathoming severe Plasmodium vivax disease . Nat Med. 2011, 17:48-9. 10.1038/nm0111-48

8. Murphy GS, Basri H, Purnomo, et al.: Vivax malaria resistant to treatment and prophylaxis with chloroquine. Lancet. 1993, 341:96-100. 10.1016/0140-6736(93)92568-e

9. Mendes C, Dias F, Figueiredo J, et al.: Duffy negative antigen is no longer a barrier to Plasmodium vivaxmolecular evidences from the African West Coast (Angola and Equatorial Guinea). PLoS Negl Trop Dis. 2011, 5:1192. 10.1371/journal.pntd.0001192

10. Mourao LC, Roma PM, Sultane Aboobacar Jda S, et al.: Anti-erythrocyte antibodies may contribute to anaemia in Plasmodium vivax malaria by decreasing red blood cell deformability and increasing erythrophagocytosis. Malar J. 2016, 15:397. 10.1186/s12936-016-1449-5

11. Ribeiro BD, Cassiano GC, de Souza RM, et al.: Polymorphisms in Plasmodium vivax circumsporozoite protein (CSP) influence parasite burden and cytokine balance in a pre-amazon endemic area from Brazil. PLoS Negl Trop Dis. 2016, 10:0004479. 10.1371/journal.pntd.0004479

12. Poirriez J, Landau I, Verhaeghe A, Savage A, Dei-Cas E: Atypical forms of Plasmodium vivax. apropos of a case. Ann Parasitol Hum Comp. 1991, 66:149-54. 10.1051/parasite/1991664149

13. Vasallo FM, Perez MS, Ortega EM, Ortega MT, Diaz MM: Atypical P. vivax and P. ovale morphology in two imported cases of malaria in Spain. Parasitologia. 1997, 39:33-5.

14. Wang YB, Kong XL, Xu Y, et al.: Large trophozoites in blood smear of falciparum malaria: one case report. (Article in Chinese). Zhongguo Xue Xi Chong Bing Fang Zhi Za Zhi. 2014, 26:355-6. 